\title{
Civilisations
}

Revue internationale d'anthropologie et de sciences

humaines

52-1 | 2004

Transmission des savoirs et interactions culturelles

\section{La transmission du savoir en Mésopotamie ancienne}

Philippe Talon

\section{OpenEdition}

Journals

Édition électronique

URL : http://journals.openedition.org/civilisations/734

DOI : $10.4000 /$ civilisations.734

ISSN : 2032-0442

\section{Éditeur}

Institut de sociologie de l'Université Libre de Bruxelles

\section{Édition imprimée}

Date de publication : 1 mai 2004

Pagination : 25-33

ISSN : 0009-8140

Référence électronique

Philippe Talon, «La transmission du savoir en Mésopotamie ancienne », Civilisations [En ligne], 52-1 | 2004, mis en ligne le 27 janvier 2009, consulté le 02 juin 2020. URL : http://journals.openedition.org/ civilisations/734; DOI : https://doi.org/10.4000/civilisations.734 


\section{La transmission du savoir en Mésopotamie ancienne}

\section{PHILIPPE TALON}

A titre de simple rappel et pour fixer quelque peu le cadre chronologique dans lequel nous allons évoluer, je rappellerai que l'écriture cunéiforme fut inventée aux alentours de $3200 \mathrm{BC}$ dans le sud de la Mésopotamie, probablement dans la ville d'Uruk ${ }^{1}$. Cette écriture fut employée, avec des variantes, dans tout le Proche-Orient ancien et servira à noter de nombreuses langues appartenant à des familles linguistiques variées : principalement le sumérien et l'akkadien, mais aussi le hittite, le hurrite, l'urartéen, l'élamite, etc.

Les derniers textes en cunéiforme sont, quant à eux, datés du début de notre ère, et, selon des travaux récents, cette écriture cesse d'être employée avec la fermeture des temples de Babylonie au début de l'empire sassanide; j'y reviendrai plus loin.

A l'origine inventée presque certainement pour répondre aux besoins de plus en plus complexes d'une administration centralisée, celle de «l'empire » commercial d'Uruk ${ }^{2}$, l'écriture va très vite être appliquée à des domaines moins profanes. Les toutes premières archives ${ }^{3}$, contemporaines de l'invention elle-même, contiennent en effet immédiatement des textes dont l'utilité ne peut être qu'intellectuelle. Il s'agit de listes de signes classées selon des principes divers : listes de noms de professions, listes de noms de vases, d'animaux, de dieux ${ }^{4}$, etc.

On a souvent classé ces listes dans le genre des textes scolaires, en imaginant qu'elles servaient à l'apprentissage des scribes. Peut-être est-ce vrai, mais la qualité de leur ductus et le nombre de copies conservées montrent qu'elles devaient également avoir une autre fonction, plus élaborée. Ces listes sumériennes furent par la suite continuellement recopiées et enrichies, pourvues de traductions akkadiennes, recompilées et reclassées selon d'autres clefs et ce, jusqu'à la disparition de l'écriture cunéiforme. Elles témoignent de l'activité intellectuelle des savants de l'époque et

1. Voir Talon-Van Lerberghe 1997 ; J.-J. Glassner, Ecrire à Sumer 2000.

2. Cf. entre autres, Lebeau 1990.

3. Green-Nissen 1987 ; Englund-Nissen 1993 ; Englund-Grégoire 1991.

4. Litke 1998. 
représentent une tentative d'organisation du cosmos fondée sur l'énumération exhaustive de ses constituants, réels ou mythiques ${ }^{5}$.

C'est là une des caractéristiques de l'esprit mésopotamien, souvent qualifié de pré-philosophique, que d'exposer une théorie ou un concept par le procédé de l'énumération. A titre d'exemple, je citerai un célèbre texte mathématique daté du XVIII ${ }^{\mathrm{e}}$ siècle qui contient toute une série de problèmes tournant autour de la résolution de l'équation du second degré. Plutôt que de nous donner la formule de résolution (fort proche d'ailleurs de la nôtre), le texte nous présente une vingtaine de problèmes et les étapes successives de leurs solutions. La solution est, dans tous les cas, la même : 30 , ce qui montre bien qu'il ne s'agit pas de problèmes réels, liés à un calcul pratique quelconque, mais l'exposé d'une méthode 6 .

Toutes ces listes remplissent aujourd'hui plus de quinze gros volumes ${ }^{7}$ et elles sont loin d'avoir été toutes publiées. Elles concernent tous les aspects de la vie intellectuelle des Mésopotamiens : listes de signes cunéiformes avec leurs valeurs, leurs noms, etc ; listes d'objets de la vie de tous les jours, une véritable encyclopédie classée par thèmes ; listes de formes morphologiques sumériennes et akkadiennes, en somme une grammaire ; listes d'observations astronomiques; listes de présages astrologiques ou autres ; listes de divinités, classées par familles et cours divines ; listes rétroactives de mots akkadiens classés par racines et traduits en sumérien ; commentaires ésotériques sur les noms des dieux, des villes, des temples, etc.

En somme, tout le savoir suméro-babylonien a été de la sorte transmis au cours des trois mille ans de l'utilisation de l'écriture cunéiforme. Cette abondante littérature ne s'éteindra qu'avec la disparition de l'outil auquel elle était indissociablement liée.

Il faut souligner que cette littérature lexicographique apparaît dès les premiers pas de l'écriture. Elle naît avec elle et meurt avec elle. Les savants mésopotamiens le savaient et en tiraient gloire. Entièrement tournés vers le passé, ils se vantaient de posséder un savoir ${ }^{8}$ qui leur venait « d'avant le Déluge », directement issu des dieux et transmis par eux au principal héros civilisateur, Adapa.

Ce personnage nous a été décrit en grec par Bérose ${ }^{9}$ (un prêtre de Bēl au IV siècle av. J.-C.) comme un être mi-homme mi-poisson, sorti du Golfe arabo-persique pour apporter la civilisation en Mésopotamie. Aucun texte autochtone ne nous parle de cela, mais nous savons que le costume en forme de poisson était l'apanage des prêtres exorcistes voués au dieu Ea, dieu de la Sagesse et de la Connaissance. Un mythe relatif à Adapa ${ }^{10}$ nous explique toutefois comment ce savoir a pu être transmis aux hommes. Au début, le dieu Ea résidait dans son palais et s'occupait de tous les travaux domestiques. Sa ville étant située au bord du Golfe Persique, tous les jours il prenait son bateau et s'en allait pêcher. Il avait créé un homme, Adapa, qui gardait le palais pendant son absence et durant son sommeil. Mais un jour, pendant le sommeil

\footnotetext{
5. Talon 1998.

6. Talon 1992.

7. MSL 1937.

8. Cf. la déclaration d'Assurbanipal citée plus loin.

9. Voir Burstein 1978.

10. Edition dans Picchioni 1981 ; Talon 1990 ; Izre'el 2001.
} 
d'Ea, Adapa prit le bateau et s'en alla pêcher. Malheureusement, il ne connaissait pas le maniement du bateau et celui-ci dériva au milieu de l'océan. Au début la mer était calme, mais voilà que se lève soudain le Vent du Sud. Il fait chavirer le bateau et Adapa se retrouve à l'eau.

Ce Vent du Sud n'est pas n'importe qui. C'est le Vent chaud du désert qui dessèche le sud de l'Iraq, c'est lui qui apporte les tempêtes de sable qui abattent les récoltes. C'est un démon puissant, le célèbre Pazuzu. Démon tellement puissant qu'on pouvait en faire des amulettes destinées à se protéger contre... les démons. En se conciliant Pazuzu, on se mettait en quelque sorte à l'abri de tous ses congénères moins terribles que lui.

Adapa est furieux d'avoir été jeté à l'eau et maudit le Vent du Sud en lui brisant les ailes. Aussitôt l'univers est frappé de stupeur. Dans les cieux, le roi des dieux, Anu, s'interroge : "Qui a brisé les ailes du Vent du Sud? » et on lui répond qu'il s'agit d'Adapa, un serviteur d'Ea. Ordre est immédiatement donné d'aller chercher le coupable.

Alors Ea, qui dans sa profonde sagesse a compris toutes les implications de la situation, va donner ses instructions à Adapa. Ses instructions sont détaillées et Adapa va les suivre à la lettre.

Il commence par revêtir un vêtement de deuil, puis se met en route pour le palais céleste. Arrivé à la porte des cieux, il salue les deux divinités qui gardent l'entrée : Dumuzi et Ningizzida. Ceux-ci l'interrogent : « Pourquoi portes-tu le deuil? » Et Adapa de répondre, selon les instructions : « Je porte le deuil de deux divinités qui sont mortes : Dumuzi et Ningizzida! » Alors les gardiens éclatent de rire et le laissent passer en promettant de parler en sa faveur.

Arrivé en présence du roi des dieux, Anu, Adapa est sommé d'expliquer pourquoi il a brisé les ailes du Vent du Sud. Il explique comment il a emprunté le bateau de son maître et a chaviré à cause du vent. C'est sous l'effet de la colère qu'il a maudit le Vent du Sud. Les dieux alors se calment et lui pardonnent.

Cependant un problème subsiste : Adapa est monté aux cieux, il a donc vu les secrets du ciel et de la terre. Il possède maintenant une connaissance qui n'est pas destinée aux hommes. Que faire? Déjà dans le mythe du Déluge, les dieux s'étaient trouvés devant un pareil dilemme avec Uta-Napištim, le Noé babylonien. Pour résoudre le problème, les dieux lui avaient donné l'immortalité et lui avaient ordonné de vivre aux confins du monde.

Anu va donc résoudre la difficulté de la même manière : puisque Adapa a vu ce que les mortels ne peuvent pas voir, qu'on lui serve la nourriture et l'eau-de-vie afin qu'il devienne immortel, qu'il devienne un dieu en quelque sorte. Et surtout qu'il ne puisse ramener sur terre cette Connaissance qu'il a acquise... Mais Adapa, suivant fidèlement les instructions de son maître Ea, refuse la Vie. Voyant cela, Anu éclate de rire. C'est une fois de plus une ruse du dieu Ea. En empêchant Adapa de devenir immortel, Ea a fait en sorte que celui-ci pourra revenir sur terre, porteur d'un savoir divin. Et Ea met ainsi en place une longue tradition de prêtres et de savants qui se transmettront cette connaissance. C'est parmi eux que se trouveront tous les grands savants, prêtres et exorcistes, tous les grands conseillers royaux, à commencer par les Sept Sages, compagnons des rois les plus antiques.

Dans ce texte, le caractère du dieu Ea transparaît bien, fidèle à tout ce que nous savons de lui par d'autres mythes. C'est un dieu prométhéen : il agit par ruse pour 
contourner les décisions des grands dieux, lorsque celles-ci sont dangereuses pour l'homme, ou lorsque les dieux veulent garder pour eux ce que lui, Ea, estime leur être destiné. C'est le cas lors du Déluge, notamment, où il trahit les intentions divines d'anéantir l'humanité en prévenant Uta-Napištim et en lui indiquant comment construire une arche ${ }^{11}$.

Il est le grand patron des médecins, des astrologues et des exorcistes, en somme de tous les détenteurs du savoir le plus caché, le plus secret. Il préside aussi au destin de ceux que nous nommons les « scribes », en réalité les grands intellectuels ou académiciens de l'époque (ummānu), ceux qui avaient maîtrisé tous les secrets de l'écriture et pouvaient en jouer comme les grands rabbins juifs jouaient de la Torah dans les Midrash $^{12}$. Ces jeux ne sont évidemment pas gratuits. Les règles de l'exégèse rabbinique, comme celles de l'interprétation babylonienne, permettent de retrouver derrière les mots et les signes d'écriture le sens caché du message divin. Ils éclairent le texte pour en révéler toutes les facettes. Lorsque l'exégèse devient mystique, comme dans la Kabbale, les interprétations qu'autorise le texte donnent au disciple les moyens de s'approcher de Dieu et d'en contempler la divinité ${ }^{13}$.

Nous retrouvons la même approche dans les textes mésopotamiens. De nombreuses tablettes, contenant des œuvres diverses, mais le plus souvent des commentaires cultuels ou ésotériques, portent un colophon interdisant leur accès au non-initié (celui qui ne sait pas) et exigeant de l'initié (celui qui sait) d'en prendre grand soin ${ }^{14}$.

Ce savoir était certainement l'apanage d'un groupe relativement restreint d'académiciens. Les lettres de l'époque néo-assyrienne nous dépeignent remarquablement les activités de ces savants et leur mode de vie et de pensée. Ils sont organisés en cinq « domaines $\rangle^{15}$ :

- țupšarrūtu art de l'écriture et astrologie

- bārūtu divination (essentiellement l'hépatoscopie)

- āšipūtu exorcisme et magie

- asūtu médecine

- kalūtu chants et lamentations

Ceux qui appartiennent à ces cinq domaines sont les Sages, les «philosophes ", dont le modèle par excellence est Adapa. Un texte administratif ${ }^{16}$ daté des environs de 650 énumère nommément 7 astrologues, 9 exorcistes, 5 devins, 9 médecins, 6 lamentateurs, 3 augures, 3 savants égyptiens (harțibì) et 3 scribes égyptiens. Il s'agit vraisemblablement là de l'ensemble du personnel académique du palais de Ninive à cette époque, en tout cas ses membres les plus importants. A la tête de chacune de ces cinq Disciplines se trouve un chef (un Grand, rabu, $c f$. le terme rabbin), mais le principal d'entre eux était sans nul doute le rab țpšarri ou rab ummāni « le Grand Scribe » ou « Grand Sage », que l'on nommait l'ummānu du roi. Celui-ci siégeait au cabinet

11. Voir la traduction de l'épisode du Déluge dans Tournay-Shaffer 1994 ; Bottéro 1992.

12. Cf. Cavigneaux 1987 ; Liebermann 1987.

13. Gruenwald 1997.

14. Borger 1971 ; Beaulieu 1992.

15. SAA X, xiii-Xv ; Parpola 1993a.

16. SAA VII $n^{\circ} 1$. 
restreint du roi, en compagnie du souverain et des 7 grands ministres, un total de 9 personnes dont dépendaient les destinées de l'empire assyrien ${ }^{17}$. Ce cabinet restreint était organisé sur le même modèle que l'assemblée des Grands Dieux, le roi jouant dans le monde matériel le rôle du maître du panthéon. Chaque ministre avait, lui aussi, sa contrepartie divine, son modèle céleste. Le Grand Académicien représentait bien sûr le dieu Ea sur terre.

Cet homme, avec son équipe de savants subordonnés à lui, était responsable de la santé physique et morale de l'empereur. Il était chargé de scruter l'univers à la recherche des messages divins porteurs de présages, mais aussi et surtout, d'y apporter la réponse adéquate. Tout présage possède en lui sa solution. Comme l'écrit Balasî, le Grand Académicien du prince héritier ${ }^{18}$, au roi : « Il y a eu un tremblement de terre. C'est mauvais signe. On doit accomplir le rituel contre les tremblements de terre et tes dieux éloigneront le mauvais présage : 'Ea l'a fait, Ea peut le défaire '. Celui qui a causé le tremblement de terre a aussi créé le rituel qui permet de s'en prémunir ». Le Grand Ummānu était en quelque sorte le gardien ou le conseiller spirituel du roi.

Tous ces grands savants, même s'ils ne siègent pas au conseil restreint, ont le privilège de pouvoir communiquer directement avec le roi. Celui-ci n'était certainement pas ignorant de toutes ces matières. Il avait reçu l'enseignement de l'ummānu lorsqu'il était prince héritier et continuait à être conseillé par le Grand Académicien. C'est surtout vrai pour un roi comme Assurbanipal, qui s'en vante dans ses inscriptions:

Marduk, le Sage des dieux, m'offrit en cadeau un vaste entendement et une large intelligence.

Nabû, le scribe universel, me donna comme présent sa connaissance de la sagesse.

Ninurta et Nergal dotèrent mon corps d'une puissance, d'une virilité et d'une force sans égales.

J'ai appris l'art du Sage Adapa : les secrets cachés, la science complète de l'écriture,

je suis à même de distinguer les signes du ciel et de la terre, je suis en mesure de délibérer dans l'assemblée des savants,

je suis capable d'analyser « Si le foie est à l'image du ciel » avec les savants expérimentés.

Je résous même les réciproques et les multiplications complexes dont on ne donne pas la solution.

Comme je peux lire les textes littéraires parfaits dont le sumérien est obscur et l'akkadien difficile à clarifier,

je suis en mesure d'examiner les stèles de pierre d'avant le Déluge, qui sont un mélange de kakku sakku ${ }^{19}$.

Voici ce qu'avec un partenaire bien choisi je faisais tous les jours :

je peux bondir de toute part sur des pur-sang, je suis capable de chevaucher de fougueux chevaux de race,

17. Parpola 1995.

18. SAA X, $\mathrm{n}^{\circ} 56$.

19. sakku signifie probablement « obscur », cf. CAD S, 78a; kakku sakku pourrait être un recueil médical ou exorcistique ou encore un objet d'une forme précise utilisée dans le rituel, cf. Parpola 1993b, p. 22. 
je sais brandir la lance, je tire des flèches, symbole martial, je lance de vibrants javelots comme de simples flèches.

Je sais tenir les rênes comme un vrai conducteur de char, je fais tourner les roues,

je brandis boucliers lourds et légers comme un maître armurier,

je suis au courant des grandes connaissances de l'ensemble de tous les savants.

Les savants se réclament généralement d'une lignée célèbre. Ils se disent descendants de grands hommes du $2^{\mathrm{e}}$ millénaire et principalement de Sîn-leqe-unnīnī, à qui on attribue la rédaction de la version finale de l'Epopée de Gilgameš. De là, la lignée arrive à Gabbu-ilāni-ēreš, Grand Académicien d'Aššurnașirpal II au IX ${ }^{\mathrm{e}}$ siècle, puis à Nabû-zuqup-kēna, l'ummānu de Sargon II et de Sennacherib vers $700^{20}$. Souvent, la transmission se fait de père en fils. Dans d'autres cas, il doit s'agir d'adoption ou de filiation « intellectuelle » plutôt que réelle.

La tradition de ces grands Savants associés au souverain s'est perpétuée jusqu'à l'époque hellénistique. Ils étaient considérés comme les émules des Sept Sages dont la légende voulait qu'ils eussent conseillé les Rois antédiluviens. Plus tard, leur souvenir apparaît encore dans les légendes araméennes, puisque l'un d'entre eux, le Grand Ummānu d'Assurbanipal21, survivra sous son nom araméen Ahiqar jusque dans des versions en slavon et en arménien, à l'époque chrétienne:

A l'époque du roi Ayalu, U'an était apkallu,

A l'époque du roi Alalgar, U'anduga était apkallu,

A l'époque du roi Ammelu'anna, Enmeduga était apkallu,

A l'époque du roi Ammegalanna, Enmegalamma était apkallu,

A l'époque du roi Enme'ušumugalanna, Enmebulugga était apkallu,

A l'époque du roi Dumuzi le Berger, Anenlilda était apkallu,

A l'époque du roi Enmeduranki, Utu'abzu était apkallu.

[Après le Déluge], pendant le règne d'Enme(r)kar, Nungalpiriggal était apkallu, [celui qu'Ištar] fit descendre des cieux dans l'Eanna. La lyre de bronze

[dont le ...] est de lapis-lazuli, œuvre de Ninagal,

[il fit. Dans le ...], séjour de ..., on plaça la lyre devant Anu.

A l'époque du roi Gilgameš, Sîn-leqe-Unnīni était ummānu, A l'époque du roi Ibbi-Sîn, Kabtu-il-Marduk était ummānu, A l'époque du roi Išbi-Erra, Sidu, alias Enlil-ibni était ummānu, A l'époque du roi Abi-Ešuh, Šū-Gula et Taqī̌s-Gula étaient ummānu, A l'époque du roi ..., Esagil-kīni-apla était ummānu,

A l'époque du roi Adad-apla-iddina, Esagil-kīni-ubba était ummānu, A l'époque du roi Nabuchodonosor, Esagil-kīni-ubba était ummānu, A l'époque du roi Assarhaddon, Aba-Enlil-dari était ummānu, celui que les Ahlaméens (Araméens) appellent Aḥiqar, [Alias?] Niqaqurušū.

20. Voir la liste des membres du cercle restreint pendant le règne des Sargonides dans SAA X, p. xxvi.

21. Aba-Enlil-dari, probablement un autre nom d'Ištar-šumu-ēreš? 
[Tablette] d'Anu-bēlšunu, fils de Nidittu-Anu, descendant de Sîn-leqe-unnīni, [prêtre kalû] d'Anu et d'Antum, citoyen d'Uruk. (Ecrit) de sa propre main.

[Uru]k, le 10 du mois Ayyāru, l'an 147. Antiochos était roi.

Celui qui révère Anu n'emportera pas cette (tablette).

Si l'on suit les théories de l'assyriologue finlandais Simo Parpola, le cabinet restreint, avec à sa tête le Roi et le Grand Ummānu, représentait, dans le monde matériel, l'assemblée des Grands Dieux, avec à sa tête les dieux Anu et $\mathrm{Ea}^{22}$. Ils étaient symbolisés dans les palais assyriens par l'Arbre de Vie, sous la forme d'un schéma que l'on retrouvera dans la Kabbale avec l'Arbre des Séfirot ${ }^{23}$. Cette construction est organisée en trois triades superposées, dont chaque point est occupé par un dieu (ou, dans la version humaine, par un ministre). Ces neuf dieux symboliseraient les neuf émanations du dieu Aššur, lui-même divinité transcendante et immanente, représenté sous la forme du Soleil Ailé, celui dont on dit qu'il est « le roi de tous les dieux, créateur de lui-même (autogène), le père des grands dieux, celui qui est exalté dans l'Abysse, le roi du ciel et de la terre, seigneur de tous les dieux, qui a engendré les Igigi et les Anunnaki, constructeur de la voûte du ciel et du fondement de la terre, créateur de l'univers, qui séjourne dans les pures constellations, le premier des dieux, celui qui décrète les destins $\gg{ }^{24}$. A la base de l'Arbre Divin se trouve le Roi, lien cosmique entre le monde divin et le monde matériel. Au centre de l'Arbre, position privilégiée par excellence, se trouve la déesse Ištar, symbolisée dans le Cabinet Royal par le Grand Eunuque.

Dans ce cadre, Ištar est réellement l'une des figures essentielles et fondamentales du panthéon assyrien. Elle est à la fois fille, sœur et mère des huit autres Grands Dieux. Elle est également l'épouse, la mère et la fille du dieu transcendant, Aššur lui-même. Elle forme en quelque sorte avec Aššur et le Dieu-Fils, Nabû, la Trinité assyrienne.

C'est elle qui s'exprime principalement par la bouche des prophètes. Ainsi un oracle adressé au roi Assarhaddon dit $\operatorname{ceci}^{25}$ :

« Ne crains rien, Assarhaddon!

Je suis Bēl (le Seigneur). En te parlant, je veille sur les poutres de ton cœur. Lorsque ta mère t'a donné naissance, 60 grands dieux se tenaient à mes côtés pour te protéger. Sîn était à ta droite, Šamaš à ta gauche. 60 grands dieux se tenaient autour de toi pour te vêtir.

Ne mets pas ta confiance en l'homme. Lève les yeux, regarde-moi.

Je suis Ištar d'Arbèles. J'ai réconcilié Aššur avec toi. Lorsque tu étais petit, je t'ai pris près de moi. Ne crains rien, révère-moi!

Où est l'ennemi qui t'a attaqué sans que je n'intervienne. L'avenir sera comme le passé. Je suis Nabû, le maître du calame. Révère-moi! »

22. Cf. Parpola 1995.

23. Parpola 1993b ; Parpola 2000.

24. SAA XII $\mathrm{n}^{\circ} 86$, lignes 7-11.

25. SAA IX, $\mathrm{n}^{\circ} 1$, col. ii, 16'-40’. $C f$. également SAAS VII. 
On voit dans ce texte remarquable, Ištar s'adresser au roi en prenant successivement la forme de Bēl (c'est-à-dire Marduk ou Aššur), d'Ištar et de Nabû : on peut y voir le prototype du Père, du Fils et du Saint Esprit dans la tradition chrétienne. Ištar est au centre de la religion assyrienne, comme elle siège au centre de l'Arbre Sacré assyrien, prototype de la Séfira que les Kabbalistes nommeront Tiféret, "Beauté ». Elle est androgyne et porte la barbe : «Comme Aššur, elle porte la barbe et est revêtue d'une aura brillante $»^{26}$.

Toujours selon Parpola, elle aurait été la figure centrale d'un culte mystique et initiatique, que l'on pourrait appeler les Mystères d'Ištar. Les initiés de ce culte, comme leurs émules plus tardifs des cultes d'Attis et de la Dea Syria, cherchaient à atteindre l'union mystique avec Dieu (ici Aššur) par son intermédiaire, en pratiquant toutes sortes de rites de purification et de mortification allant jusqu'à l'émasculation volontaire. De là le rôle prééminent joué par les eunuques à la cour des rois d'Assyrie.

Dans ce cadre, qui demande à être encore affiné et confirmé en partie, la transmission de la Connaissance joue bien évidemment un rôle central ${ }^{27}$. Tout le canon de la littérature scientifique, mais aussi les grandes œuvres littéraires, comme l'Epopée de Gilgame $\breve{s}^{28}$ ou la Descente d'Ištar aux Enfers, font partie intégrante des Mystères assyriens. On comprend mieux la motivation extraordinaire des savants à copier et à recopier ces textes qui leur étaient profondément sacrés, car ils contenaient l'exposé de la Voie menant à la Divinité.

C'est probablement surtout vrai de la Descente d' šstar $^{29}$, dont les parallèles avec le mythe gnostique chrétien de l'Exégèse de l'Ame laissent supposer qu'il représentait la Descente de la Déesse, prototype de l'Ame Cosmique ou de la Sophia, dans le monde matériel et sa renaissance ensuite, suivie de la réunion avec son père, le Créateur. En pénétrant dans le monde matériel, Ištar apporte avec elle la Connaissance. Le mythe est certainement lié à la continuité et à la renaissance du pouvoir royal, lien par excellence entre les mondes humain et divin. Lorsque la déesse remonte des Enfers, image du monde matériel, le texte dit que remontent avec elle les insignes du pouvoir, lesquels sont ainsi transcendés et unis avec Dieu, renouvelés à l'infini.

Il reste à prouver que tous ces liens qui se tissent, semble-t-il, avec les religions et les philosophies postérieures à l'empire assyrien sont bien réels et non le produit de l'imagination de certains. Mais de trop nombreuses coïncidences permettent d'entrevoir comment la longue tradition mésopotamienne a pu se perpétuer dans le monde hellénistique, puis dans les conceptions judaïques, gnostiques ou chrétiennes.

Lorsque l'empire assyrien s'effondra en 612, sous les coups des Mèdes et des Babyloniens coalisés, les Assyriens se regroupèrent un moment en Mésopotamie du Nord, à Harrān, espérant une intervention égyptienne qui vint trop tard. Néanmoins, Harrān resta un grand centre intellectuel par la suite. Nabonide, le dernier roi de la dynastie néo-babylonienne y entreprit de grands travaux. Plus tard, les philosophes païens des premiers siècles de notre ère y venaient en pèlerinage. Même l'empereur Julien vint y visiter une école néo-platonicienne dont on sait qu'elle ne disparut qu'au

26. SAA III, $\mathrm{n}^{\circ} 7$, ligne 6 .

27. Cf. Talon (sous presse).

28. Cf. Parpola 1998.

29. Talon 1988. 
$\mathrm{X}^{\mathrm{e}}$ siècle $^{30}$. Des philosophes néo-platoniciens comme Damascius, au $\mathrm{VI}^{\mathrm{e}}$ siècle, connaissaient encore remarquablement bien certaines grandes œuvres mésopotamiennes, comme le Mythe de la Création ${ }^{31}$.

Jusque vers 250 de notre ère, on a perpétué en Babylonie l'usage de l'écriture cunéiforme. On tente, à cette époque, de noter en grec les langues sumérienne et akkadienne, comme l'attestent une série de tablettes ${ }^{32}$. C'est probablement lorsque les Sassanides ont définitivement fermé les temples babyloniens que s'est enfin éteinte la tradition autochtone, comme cela s'est passé un peu plus tard en Egypte.

Cessant d'écrire en cunéiforme, perdant le lien avec les sources directes, les tenants de cette très ancienne philosophie ont encore maintenu leur enseignement dans le monde proche-oriental et méditerranéen pendant quelques siècles, mais, devant la montée du christianisme, les doctrines païennes ont lentement mais sûrement reculé. Elles restent encore détectables dans les écrits de la Kabbale, dont les premiers textes apparaissent précisément au XII ${ }^{\mathrm{e}}$ siècle. A travers le prisme très différent de la Kabbale, les conceptions assyriennes, toujours revues, enrichies et complétées, réinterprétées sont peut-être bien arrivées jusqu'à nous.

Philippe Talon, Professeur à l'Université Libre de Bruxelles.

30. Cf. Chuvin 1991 ; Tardieu 1990 ; Green 1992.

31. Cf. Talon (sous presse).

32. Voir Geller 1997. 


\section{RÉFÉRENCES BIBLIOGRAPHIQUES}

BEAULIEU P.-A.

1992 New Light on Secret Knowledge in Late Babylonian Culture, Zeitschrift für Assyriologie 82 : 98111.

BORGER R.

1971 Geheimwissen, Reallexikon der Assyriologie 3 : 188-191.

BOTTERO J.

1992 L'Epopée de Gilgameš. Le grand homme qui ne voulait pas mourir, Paris : Gallimard : 295 p.

BURSTEIN S.M.

1978 The Babyloniaca of Berossus, Studies on the Ancient Near East I/5. Malibu : Undena Publications : 39 p.

CAD Chicago Assyrian Dictionary.

CAVIGNEAUX A.

1987 Aux sources du Midrash : l’herméneutique babylonienne, Aula Orientalis 5 : 243-255.

CHUVIN P.

1991 Chronique des derniers païens, Paris : Les Belles Lettres/Fayard : 350 p.

ENGLUND R.K., GREGOIRE J.-P.

1991 The Proto-Cuneiform Texts from Djemdet Nasr, Materialen zu den frühen Schriftzeugnissen des Vorderen Orients I, Berlin : Gebr. Mann Verlag : 220 p.

ENGLUND R.K., NISSEN H.J.

1993 Die lexikalischen Listen der archaischen Texte aus Uruk, Archaische Texte aus Uruk 3, Berlin : Gebr. Mann Verlag : 327 p.

GELLER M.J.

1997 The Last Wedge, Zeitschrift für Assyriologie 87 : 43-95.

GLASSNER J.-J.

2000 Ecrire à Sumer, Paris : Seuil, L’Univers Historique : 302 p.

GREEN T.M.

1992 The City of the Moon God. Religious Traditions of Harran, Leiden-New York : Ed. E.J. Brill : $232 \mathrm{p}$.

GREEN M.W., NISSEN H.J.

1987 Zeichenliste der archaischen Texte aus Uruk. Archaische Texte aus Uruk 2, Berlin : Gebr. Mann Verlag : $377 \mathrm{p}$.

GRUENWALD I.

1997 How Much Qabbalah in Ancient Assyria? in PARPOLA S., WHITING R.M. (éds), Assyria 1995. Proceedings of the 10th Anniversary Symposium of the Neo-Assyrian Text Corpus Project, Helsinki, September 7-11. Helsinki : Neo-Assyrian Text Corpus Project : 115-128.

IZRE'EL S.

2001 Adapa and the South Wind. Language has the Power of Life and Death, Mesopotamian Civilization 10, Winona Lake, Ind. : Eisenbrauns : 183 p.

LEBEAU M.

1990 Esquisse d'une histoire de la Haute Mésopotamie aux débuts de l'Age du Bronze, Zeitschrift für Assyriologie 80 : 241-296. 


\section{LIEBERMANN S.J.}

1987 A Mesopotamian Background for the So-Called Aggadic « Measures » of Biblical Hermeneutics?, Hebrew University College Annual 58 : 157-225.

\section{LITKE R.L.}

1998 A Reconstruction of the Assyro-Babylonian God-Lists, AN : da-nu-um and AN : Anu ša amēli, New Haven Yale Babylonian Collection $3: 281$ p.

MSL

1937 Materialen zum Sumerischen Lexikon, Rome, 1937-.

PARPOLA S.

1993a Mesopotamian Astrology and Astronomy as Domains of the Mesopotamian «Wisdom », in GALTER H.D., Die Rolle der Astronomie in den Kulturen Mesopotamiens, Grazer Morgenländische Studien $3: 47-60$.

1993b The Assyrian Tree of Life : Tracing the Origins of Jewish Monotheism and Greek Philosophy, Journal of Near Eastern Studies 52 : 161-208.

1995 The Assyrian Cabinet, in DIETRICH M. et LORETZ O. (éds), Vom Alten Orient zum Alten Testament : Festschrift für Wolfram Freiherrn von Soden zum 85, Geburtstag am 19. Juni 1993, Alter Orient und Altes Testament $240: 379-401$.

1998 The Esoteric Meaning of the Name of Gilgamesh, in PROSECKY J. (éd.), Intellectual Life of the Ancient Near East. Papers Presented at the 43rd Rencontre Assyriologique Internationale, Prague, July 1-5, 1996, Prague : Oriental Institute : 315-330.

2000 Monotheism in Ancient Assyria, in PORTER B.N. (éd.), One God or Many? Concepts of Divinity in the Ancient World, Transactions of the Casco Bay Assyriological Institute I : 165-210.

\section{PICCHIONI S.}

1981 Il Poemetto di Adapa, Budapest : Az Eötvös Loránd Tudományegyetem 27 : 177 p.

SAA State Archives of Assyria

SAA III LIVINGSTONE A.

1989 Court Poetry and Literary Miscellanea, State Archives of Assyria III. Helsinki : Helsinki University Press : $183 \mathrm{p}$.

SAA VII FALES F.M., POSTGATE J.N.

1992 Imperial Administrative Records, Part I Palace and Temple administration, State Archives of Assyria VII, Helsinki : Helsinki University Press : 260 p.

SAA IX PARPOLA S.

1997 Assyrian Prophecies, State Archives of Assyria IX. Helsinki : Helsinki University Press : 84 p.

SAA X PARPOLAS.

1993 Letters from Assyrian and Babylonian Scholars, State Archives of Assyria X. Helsinki : Helsinki University Press : $421 \mathrm{p}$.

SAA XII KATAJA L., WHITING R.

1995 Grants, Decrees and Gifts of the Neo-Assyrian Period, State Archives of Assyria XII, Helsinki : Helsinki University Press : 174 p.

SAAS State Archives of Assyria Studies

\section{SAAS VII NISSINEN M.}

1998 Reference to Prophecy in Neo-Assyrian Sources, State Archives of Assyria Studies VII, Helsinki : Neo-Assyrian Text Corpus Project : 194 p.

TALON Ph.

(sous presse). Enuma Eliš and the Transmission of Babylonian Cosmology to the West, in PARPOLA S. 
(éd.), Mythology and Mythologies : Methodological Approaches to Intercultural Influences, Melammu 2, Helsinki : (à paraître).

1988 Le mythe de la Descente d'Ishtar aux Enfers, Akkadica $60: 15-25$.

1990 Le mythe d'Adapa, Studi Epigrafici et Linguistici sul Vicino Oriente 7 : 43-57.

1992 Introduction aux mathématiques babyloniennes, in MAWET Fr., TALON Ph. (éds), D'Imhotep à Copernic. Astronomie et mathématiques des origines orientales au moyen âge, Leuven : Lettres Orientales $2: 13-22$.

1998 La parole et l'écrit dans l'idéologie assyrienne, in BROZE M., DECHARNEUX B., JESPERS Ph., JONCKERS D. (éds), Oralité et Ecriture dans la Pratique du Mythe, Civilisations XLVI/1-2 : 43-64.

TALON Ph., VAN LERBERGHE K. (éds),

1997 En Syrie. Aux origines de l'écriture, Turnhout : Brepols : 302 p.

TARDIEU M.

1990 Les paysages reliques. Routes et haltes syriennes d'Isidore à Simplicius, Leuven : Peeters : 209 p.

TOURNAY R.J., SHAFFER A.

1994 L'Epopée de Gilgamesh, Littératures Anciennes du Proche-Orient 15, Paris : Le Cerf : 320 p. 